Pacific

Journal of

Mathematics

TWO CLASSES OF PSEUDOSYMMETRIC CONTACT METRIC 3-MANIFOLDS

Florence Gouli-ANDReou AND EVAGgelia Moutafi

Volume 239 No. 1

January 2009 


\title{
TWO CLASSES OF PSEUDOSYMMETRIC CONTACT METRIC 3-MANIFOLDS
}

\author{
Florence Gouli-Andreou And Evaggelia Moutafi
}

\begin{abstract}
We classify the pseudosymmetric contact metric 3-manifolds that satisfy $\nabla_{\xi} \tau=0$, and also the pseudosymmetric contact metric 3-manifolds of constant type satisfying $\nabla_{\xi} \tau=2 a \tau \phi$, where $a$ is a smooth function.
\end{abstract}

\section{Introduction}

According to R. Deszcz [1992], a Riemannian manifold $\left(M^{m}, g\right)$ is pseudosymmetric if the curvature tensor $R$ satisfies the condition $R(X, Y) \cdot R=L\{(X \wedge Y) \cdot R\}$, where $L$ is a smooth function, the endomorphism field $X \wedge Y$ is defined by

$$
(X \wedge Y) Z=g(Y, Z) X-g(Z, X) Y
$$

for all vectors fields $X, Y, Z$ on $M$, and the dot means that $R(X, Y)$ and $X \wedge Y$ act as derivations on $R$.

If $L$ is constant, $M$ said to be a pseudosymmetric manifold of constant type; if $L=0$, then $M$ is a semisymmetric manifold. Hence a pseudosymmetric manifold is a natural generalization of a semisymmetric manifold [Szabó 1982; 1985], which in turn is a generalization of a locally symmetric space, that is, one with $\nabla R=0$; see [Takagi 1972].

Three-dimensional pseudosymmetric spaces of constant type have been studied by many researchers, beginning with O. Kowalski and M. Sekizawa [1996b; 1996a; 1997; 1998]. Later, N. Hashimoto and M. Sekizawa classified 3-dimensional, conformally flat pseudosymmetric spaces of constant type [2000], while G. Calvaruso gave the complete classification of conformally flat pseudosymmetric spaces of constant type for dimensions greater than two [2006]. J. T. Cho and J. Inoguchi studied pseudosymmetric contact homogeneous 3-manifolds [2005].

It is well known that in the geometry of a contact metric manifold, the tensors $\tau=L_{\xi} g$ and $\nabla_{\xi} \tau$, introduced by S. S. Chern and R. S. Hamilton [1985], play a fundamental role. The condition $\nabla_{\xi} \tau=2 a \tau \phi$, where $a$ is a constant and $(\tau \phi)(X, Y)$ is

MSC2000: primary 53D10, 53C25, 53C15; secondary 53C 35.

Keywords: contact metric 3-manifolds, pseudosymmetric manifolds. 
interpreted as $\tau(\phi X, Y)$, is necessary for a contact metric 3-manifold to be homogeneous. We call a 3-dimensional contact metric manifold a 3- $\tau$-a manifold if it satisfies $\nabla_{\xi} \tau=2 a \tau \phi$, where $a$ is a smooth function; if $a=0$, we call it a 3- $\tau$ manifold. The condition $\nabla_{\xi} \tau=0$ appeared first in [Chern and Hamilton 1985] in the study of compact contact 3-manifolds, while Perrone [1990] proved that it is the critical point condition for the functional "integral of the scalar curvature" defined on the set of all metrics associated to the fixed contact form $\eta$. Moreover, this condition $\nabla_{\xi} \tau=0$ is equivalent to the condition requiring equality of the sectional curvature of all planes at a given point and perpendicular to the contact distribution [Gouli-Andreou and Xenos 1998a].

This article studies contact metric 3-manifolds in which

(i) $M$ is a pseudosymmetric manifold and $\nabla_{\xi} \tau=0$, where $\tau=L_{\xi} g$; or

(ii) $M$ is a pseudosymmetric manifold of constant type with $\nabla_{\xi} \tau=2 a \tau \phi$, where $a$ is a smooth function on $M$.

\section{Preliminaries}

Let $\left(M^{m}, g\right)$ for $m \geq 3$ be a connected Riemannian smooth manifold. We denote by $\nabla$ the Levi-Civita connection of $M^{m}$ and by $R$ the corresponding Riemannian curvature tensor given by $R(X, Y)=\left[\nabla_{X}, \nabla_{Y}\right]-\nabla_{[X, Y]}$.

A Riemannian manifold $\left(M^{m}, g\right)$ for $m \geq 3$ is said to be pseudosymmetric in the sense of R. Deszcz [1992] if at every point of $M$ the curvature tensor satisfies the equation

$$
(R(X, Y) \cdot R)\left(X_{1}, X_{2}, X_{3}\right)=L\left\{((X \wedge Y) \cdot R)\left(X_{1}, X_{2}, X_{3}\right)\right\},
$$

where

$$
\begin{aligned}
& (R(X, Y) \cdot R)\left(X_{1}, X_{2}, X_{3}\right)= \\
& R(X, Y)\left(R\left(X_{1}, X_{2}\right) X_{3}\right)-R\left(R(X, Y) X_{1}, X_{2}\right) X_{3} \\
& -R\left(X_{1}, R(X, Y) X_{2}\right) X_{3}-R\left(X_{1}, X_{2}\right)\left(R(X, Y) X_{3}\right)
\end{aligned}
$$

$$
\begin{aligned}
& ((X \wedge Y) \cdot R)\left(X_{1}, X_{2}, X_{3}\right)= \\
& (X \wedge Y)\left(R\left(X_{1}, X_{2}\right) X_{3}\right)-R\left((X \wedge Y) X_{1}, X_{2}\right) X_{3} \\
& \quad-R\left(X_{1},(X \wedge Y) X_{2}\right) X_{3}-R\left(X_{1}, X_{2}\right)\left((X \wedge Y) X_{3}\right),
\end{aligned}
$$

and $X \wedge Y$ is given by (1-1). In particular, if $L=0$, then $M$ is semisymmetric. For details and examples of pseudosymmetric manifolds, see [Belkhelfa et al. 2002] and [Deszcz 1992].

A contact manifold is a differentiable manifold $M^{2 n+1}$ together with a global 1 -form $\eta$ (a contact form) such that $\eta \wedge(d \eta)^{n} \neq 0$ everywhere. Since $d \eta$ is of rank $2 n$, there exists a unique vector field $\xi$ on $M^{2 n+1}$ (the Reeb or characteristic 
vector field of the contact structure $\eta)$ satisfying $\eta(\xi)=1$ and $d \eta(\xi, X)=0$ for all $X$. The distribution defined by the subspace $X \in T_{p} M: \eta(X)=0$ for $p \in M$ is called a contact distribution. Every contact manifold has an underlying almost contact structure $(\eta, \phi, \xi)$, where $\phi$ is a global tensor field of type $(1,1)$, such that

$$
\eta(\xi)=1, \quad \phi \xi=0, \quad \eta \circ \phi=0, \quad \phi^{2}=-I+\eta \otimes \xi .
$$

A Riemannian metric $g$ (the associated metric) can be defined such that

$$
\eta(X)=g(X, \xi) \quad \text { and } \quad d \eta(X, Y)=g(X, \phi Y)
$$

for all vector fields $X$ and $Y$ on $M^{2 n+1}$. We note that $g$ and $\phi$ are not unique for a given contact form $\eta$, but $g$ and $\phi$ are canonically related to each other. We refer to $\left(M^{2 n+1}, \eta, \xi, \phi, g\right)$ as a contact metric structure.

We denote by $S$ the Ricci tensor of type $(0,2)$, by $Q$ the corresponding Ricci operator satisfying $g(Q X, Y)=S(X, Y)$, and by $r=\operatorname{Tr} Q$ the scalar curvature. We also define the tensor fields $l, h$ and $\tau$ by the relations

$$
l=R(\cdot, \xi) \xi, \quad h=\frac{1}{2} L_{\xi} \phi, \quad \tau=L_{\xi} g,
$$

where $L$ is the Lie differentiation. On every contact metric manifold $M^{2 n+1}$, we have the important formulas

$$
\begin{aligned}
& h \xi=l \xi=0, \quad \eta \circ h=0, \quad \operatorname{Tr} h=\operatorname{Tr} h \phi=0, \quad h \phi=-\phi h, \\
& h X=\lambda X \quad \text { implies } \quad h \phi X=-\lambda \phi X, \\
& \nabla_{\xi} \phi=0, \quad \nabla_{X} \xi=-\phi X-\phi h X, \quad \operatorname{Tr} l=g(Q \xi, \xi)=2 n-\operatorname{Tr} h^{2}, \\
& \tau=2 g(\phi \cdot, h \cdot), \quad \nabla_{\xi} \tau=2 g\left(\phi \cdot, \nabla_{\xi} h \cdot\right) .
\end{aligned}
$$

A contact metric manifold $M^{2 n+1}(\phi, \xi, \eta, g)$ for which $\xi$ is a Killing vector field, that is, for which $L_{\xi} g=0$, is called a K-contact manifold. A contact metric manifold is $\mathrm{K}$-contact if and only if $\tau=0$ (or equivalently $h=0$ ).

If we take the product $M^{2 n+1} \times \mathbb{R}$, then the contact structure on $M^{2 n+1}$ gives rise to an almost complex structure $J$ on $M^{2 n+1} \times \mathbb{R}$ given by

$$
J\left(X, f \frac{d}{d t}\right)=\left(\phi X-f \xi, \eta(X) \frac{d}{d t}\right) .
$$

If this structure is integrable, then the contact structure is said to be normal and $M^{2 n+1}$ is called Sasakian. A contact metric manifold is Sasakian if and only if $R(X, Y) \xi=\eta(Y) X-\eta(X) Y$ for all vectors fields $X, Y$ on the manifold. If $\operatorname{dim} M^{2 n+1}=3$ then a K-contact manifold is Sasakian and the converse also holds. More details on contact manifolds are found in [Blair 2002].

Let $(M, \phi, \xi, \eta, g)$ be a contact metric 3-manifold. Let $U$ be the open subset of points $p \in M$ such that $h \neq 0$ in a neighborhood of $p$. Let $U_{0}$ be the open subset of points $p \in M$ such that $h=0$ in a neighborhood of $p$. That $h$ is a smooth function 
on $M$ implies $U \cup U_{0}$ is an open and dense subset of $M$, so any property satisfied in $U_{0} \cup U$ is also satisfied in $M$. For any point $p \in U \cup U_{0}$, there exists a local orthonormal basis $\{e, \phi e, \xi\}$ of smooth eigenvectors of $h$ in a neighborhood of $p$ (this we call a $\phi$-basis). On $U$, we put $h e=\lambda e$, where $\lambda$ is a nonvanishing smooth function assumed to be positive. From (2-8) we have $h \phi e=-\lambda \phi e$.

Lemma 2.1 [Calvaruso and Perrone 2002; Gouli-Andreou and Xenos 1998a]. On the open set $U$ we have

$$
\begin{aligned}
& \nabla_{\xi} e=a \phi e, \quad \nabla_{e} e=b \phi e, \quad \nabla_{\phi e} e=-c \phi e+(\lambda-1) \xi, \\
& \nabla_{\xi} \phi e=-a e, \quad \nabla_{e} \phi e=-b e+(1+\lambda) \xi, \quad \nabla_{\phi e} \phi e=c e, \\
& \nabla_{\xi} \xi=0, \quad \nabla_{e} \xi=-(1+\lambda) \phi e, \quad \nabla_{\phi e} \xi=(1-\lambda) e,
\end{aligned}
$$

$(2-11) \quad \nabla_{\xi} h=-2 a h \phi+(\xi \cdot \lambda) s$

where a is a smooth function,

$$
\begin{array}{ll}
b=\frac{1}{2 \lambda}((\phi e \cdot \lambda)+A) & \text { with } A=\eta(Q e)=S(\xi, e), \\
c=\frac{1}{2 \lambda}((e \cdot \lambda)+B) & \text { with } B=\eta(Q \phi e)=S(\xi, \phi e),
\end{array}
$$

and $s$ is the type $(1,1)$ tensor field defined by $s \xi=0, s e=e$ and $s \phi e=-\phi e$.

From Lemma 2.1 and the formula $[X, Y]=\nabla_{X} Y-\nabla_{Y} X$, we can prove that

$$
\begin{gathered}
{[e, \phi e]=\nabla_{e} \phi e-\nabla_{\phi e} e=-b e+c \phi e+2 \xi,} \\
{[e, \xi]=\nabla_{e} \xi-\nabla_{\xi} e=-(a+\lambda+1) \phi e,} \\
{[\phi e, \xi]=\nabla_{\phi e} \xi-\nabla_{\xi} \phi e=(a-\lambda+1) e,}
\end{gathered}
$$

and from (1-1) we estimate

$$
\begin{array}{rlrl}
(e \wedge \phi e) e & =-\phi e, & (e \wedge \xi) e=-\xi, & (\phi e \wedge \xi) \xi=\phi e, \\
(e \wedge \phi e) \phi e=e, & (e \wedge \xi) \xi=e, & (\phi e \wedge \xi) \phi e=-\xi,
\end{array}
$$

while $(X \wedge Y) Z=0$ whenever $X \neq Y \neq Z \neq X$ and $X, Y, Z \in\{e, \phi e, \xi\}$.

By direct computations, we calculate the nonvanishing independent components of the type $(1,3)$ Riemannian curvature tensor field $R$ :

$$
\begin{aligned}
R(\xi, e) \xi & =-I e-Z \phi e, & R(e, \phi e) e & =-C \phi e-B \xi, \\
R(\xi, \phi e) \xi & =-Z e-D \phi e, & R(\xi, e) \phi e & =-K e+Z \xi \\
R(e, \phi e) \xi & =B e-A \phi e, & R(\xi, \phi e) \phi e & =H e+D \xi \\
R(\xi, e) e & =K \phi e+I \xi, & R(e, \phi e) \phi e & =C e+A \xi \\
R(\xi, \phi e) e & =-H \phi e+Z \xi, & &
\end{aligned}
$$


where

$$
\begin{aligned}
C & =-b^{2}-c^{2}+\lambda^{2}-1+2 a+(e \cdot c)+(\phi e \cdot b), & & Z=\xi \cdot \lambda, \\
H & =b(\lambda-a-1)+(\xi \cdot c)+(\phi e \cdot a), & I & =-2 a \lambda-\lambda^{2}+1, \\
K & =c(\lambda+a+1)+(\xi \cdot b)-(e \cdot a), & D & =2 a \lambda-\lambda^{2}+1 .
\end{aligned}
$$

Setting $X=e, Y=\phi e$ and $Z=\xi$ in the Jacobi identity $[[X, Y], Z]+[[Y, Z], X]+$ $[[Z, X], Y]=0$ and using (2-13), we get

$$
\begin{array}{r}
b(a+\lambda+1)-(\xi \cdot c)-(\phi e \cdot \lambda)-(\phi e \cdot a)=0, \\
c(a-\lambda+1)+(\xi \cdot b)+(e \cdot \lambda)-(e \cdot a)=0,
\end{array}
$$

or equivalently $A=H$ and $B=K$.

We give the components of the Ricci operator $Q$ with respect to a $\phi$-basis:

$$
\begin{aligned}
Q e & =\left(\frac{1}{2} r-1+\lambda^{2}-2 a \lambda\right) e+Z \phi e+A \xi, \\
Q \phi e & =Z e+\left(\frac{1}{2} r-1+\lambda^{2}+2 a \lambda\right) \phi e+B \xi, \\
Q \xi & =A e+B \phi e+2\left(1-\lambda^{2}\right) \xi,
\end{aligned}
$$

where

$$
r=\operatorname{Tr} Q=2\left(1-\lambda^{2}-b^{2}-c^{2}+2 a+(e \cdot c)+(\phi e \cdot b)\right) .
$$

The relations (2-16) and (2-19) yield

$$
C=-b^{2}-c^{2}+\lambda^{2}-1+2 a+(e \cdot c)+(\phi e \cdot b)=2 \lambda^{2}-2+r / 2
$$

Definition 2.2 [Gouli-Andreou et al. 2008]. Let $M^{3}$ be a 3-dimensional contact metric manifold. Let $h=\lambda h^{+}-\lambda h^{-}$be the spectral decomposition of $h$ on $U$. If

$$
\nabla_{h^{-} X} h^{-} X=\left[\xi, h^{+} X\right]
$$

for all vector fields $X$ on $M^{3}$ and all points of an open subset $W$ of $U$, and if $h=0$ on the points of $M^{3}$ that do not belong to $W$, then the manifold is said to be a semi-K contact manifold.

From Lemma 2.1 and the relations (2-13), the condition above for $X=e$ leads to $[\xi, e]=0$; for $X=\phi e$ it leads to $\nabla_{\phi e} \phi e=0$. Hence on a semi-K contact manifold we have $a+\lambda+1=c=0$. If we apply the deformation $e \rightarrow \phi e, \phi e \rightarrow e$, $\xi \rightarrow-\xi, \lambda \rightarrow-\lambda, b \rightarrow c$ and $c \rightarrow b$, then the contact metric structure remains the same. Hence the condition for a 3-dimensional contact metric manifold to be semi-K contact is equivalent to $a-\lambda+1=b=0$.

Remark 2.3. If $M^{3}=U_{0}$ (as in [Gouli-Andreou and Xenos 1998b]), Lemma 2.1 is expressed in a similar form, where $\lambda=0, e$ is a unit vector field belonging to 
the contact distribution, the Equation (2-11) is identically zero, and the functions $A, B, D, H, I, K$ and $Z$ satisfy

$$
A=B=Z=H=K=0, \quad I=D=1, \quad C=\frac{1}{2} r-2 .
$$

Proposition 2.4. For a 3-dimensional contact metric manifold, we have

(2-21) $Q \phi=\phi Q \quad$ if and only if $\xi \cdot \lambda=2 b \lambda-(\phi e \cdot \lambda)=2 c \lambda-(e \cdot \lambda)=a \lambda=0$.

Proof. By (2-4), (2-12), (2-16) and (2-20), the relations (2-18) yield

$$
\begin{aligned}
(Q \phi-\phi Q) e & =2 Z e+4 a \lambda \phi e+B \xi \\
(Q \phi-\phi Q) \phi e & =4 a \lambda e-2 Z \phi e-A \xi \\
(Q \phi-\phi Q) \xi & =B e-A \phi e .
\end{aligned}
$$

Proposition 2.4 follows immediately.

\section{Pseudosymmetric contact metric 3-manifolds}

Let $(M, \eta, g, \phi, \xi)$ be a contact metric 3-manifold. In the case $M=U_{0}$, that is, when $(\xi, \eta, \phi, g)$ is a Sasakian structure, $M$ is a pseudosymmetric space of constant type [Cho and Inoguchi 2005]. Next, assume that $U$ is not empty, and let $\{e, \phi e, \xi\}$ be a $\phi$-basis as in Lemma 2.1.

Lemma 3.1. A contact metric 3-manifold $(M, \eta, g, \phi, \xi)$ is pseudosymmetric if and only if

$$
\begin{aligned}
B(\xi \cdot \lambda)+\left(-2 a \lambda-\lambda^{2}+1\right) A & =L A, \\
A(\xi \cdot \lambda)+\left(2 a \lambda-\lambda^{2}+1\right) B & =L B, \\
(\xi \cdot \lambda)\left(\frac{1}{2} r+2 \lambda^{2}-2\right)+A B & =L(\xi \cdot \lambda),
\end{aligned}
$$

and

$$
\begin{aligned}
A^{2}-|(\xi \cdot \lambda)|^{2}+\left(2 a \lambda-\lambda^{2}+1\right)\left(-2 a \lambda-3 \lambda^{2}+\right. & \left.3-\frac{1}{2} r\right) \\
& =L\left(-2 a \lambda-3 \lambda^{2}+3-\frac{1}{2} r\right), \\
B^{2}-|(\xi \cdot \lambda)|^{2}+\left(-2 a \lambda-\lambda^{2}+1\right)\left(2 a \lambda-3 \lambda^{2}+\right. & \left.3-\frac{1}{2} r\right) \\
& =L\left(2 a \lambda-3 \lambda^{2}+3-\frac{1}{2} r\right)
\end{aligned}
$$

where $L$ is the function in the pseudosymmetry definition (2-1).

Proof. Setting $X_{1}=e, X_{2}=\phi e$ and $X_{3}=\xi$ in Equation (2-1), we obtain

$$
(R(X, Y) \cdot R)(e, \phi e, \xi)=L(((X \wedge Y) \cdot R)(e, \phi e, \xi)) .
$$

First we set $X=e$ and $Y=\phi e$. By virtue of (2-2), (2-3), (2-14) and (2-15) we obtain

$\left(B(\xi \cdot \lambda)+\left(-2 a \lambda-\lambda^{2}+1\right) A\right) e+\left(A(\xi \cdot \lambda)+\left(2 a \lambda-\lambda^{2}+1\right) B\right) \phi e=L(A e+B \phi e)$, 
from which the first two equations of the lemma follow at once.

Similarly, setting $X=\phi e$ and $Y=\xi$ we obtain

$$
\begin{aligned}
& \left(A^{2}-|(\xi \cdot \lambda)|^{2}+\left(2 a \lambda-\lambda^{2}+1\right)\left(-2 a \lambda-3 \lambda^{2}+2-\frac{1}{2} r\right)\right) e \\
& \quad+\left((\xi \cdot \lambda)\left(\frac{1}{2} r+2 \lambda^{2}-2\right)+A B\right) \phi e=L\left(\left(-2 a \lambda-3 \lambda^{2}+2-\frac{1}{2} r\right) e+(\xi \cdot \lambda) \phi e\right),
\end{aligned}
$$

from which we get the next two equations.

Finally, setting $X=e$ and $Y=\xi$ we have

$$
\begin{aligned}
\left(B^{2}-\mid\right. & \left.|(\xi \cdot \lambda)|^{2}+\left(-2 a \lambda-\lambda^{2}+1\right)\left(2 a \lambda-3 \lambda^{2}+2-\frac{1}{2} r\right)\right) \phi e \\
& +\left((\xi \cdot \lambda)\left(\frac{1}{2} r+2 \lambda^{2}-2\right)+A B\right) e=L\left(\left(2 a \lambda-3 \lambda^{2}+2-\frac{1}{2} r\right) \phi e+(\xi \cdot \lambda) e\right),
\end{aligned}
$$

from which we obtain the last equation.

Using Equations (2-16) and (2-20), the five equations take the more convenient form

$$
\begin{array}{rlrl}
Z B+I A & =L A, & \\
Z A+D B & =L B, & & A^{2}-Z^{2}+D(I-C)=L(I-C), \\
Z C+A B & =L Z, & B^{2}-Z^{2}+I(D-C) & =L(D-C) .
\end{array}
$$

Remark 3.2. If $L=0$, the manifold is semisymmetric and (3-1) is in accordance with [Calvaruso and Perrone 2002, Equations (3.1)-(3.5)].

Proposition 3.3. Let $M^{3}$ be a 3-dimensional contact metric manifold satisfying $Q \phi=\phi Q$. Then $M^{3}$ is a pseudosymmetric space of constant type.

Proof. In [2005], Cho and Inoguchi have proved that contact metric 3-manifolds that satisfy $Q \phi=\phi Q$ are pseudosymmetric. We can improve their result by proving that these manifolds are also pseudosymmetric of constant type. We know from [Blair et al. 1990] that for these manifolds the Ricci operator has the form $Q X=\alpha X+\beta \eta(X) \xi$ or equivalently the Ricci tensor is given by the equation

$$
S=\alpha g+\beta \eta \otimes \eta,
$$

where $\alpha=(r-\operatorname{Tr} l) / 2, \quad \beta=(3 \operatorname{Tr} l-r) / 2$ and the $\phi$-sectional curvature and $\operatorname{Tr} l$ are both constant functions. Also, from [Koufogiorgos 1995] we have that the $\phi$-sectional curvature is given by the equation $r / 2-\operatorname{Tr} l$, and hence in contact metric 3-manifolds with $Q \phi=\phi Q$, the function $r=\operatorname{Tr} Q$ is also constant; obviously the functions $\alpha$ and $\beta$ in (3-2) are constant as well. The manifold is quasi-Einstein and hence pseudosymmetric, and because $\beta$ is constant it is pseudosymmetric of constant type, that is, $L$ is constant; see also [Cho and Inoguchi 2005].

Remark 3.4. If the manifold $M^{3}$ is Sasakian, we know from Cho and Inoguchi [2005] that $M^{3}$ is a pseudosymmetric space of constant type. Also, by using Remark 2.3, the system (3-1) is reduced to the equation $(C-1)(L-1)=0$. Hence 
a Sasakian 3-manifold satisfying the condition $R(X, Y) \cdot R=L((X \wedge Y) \cdot R)$ with $L \neq 1$ is a space of constant scalar curvature $r=6$, where $L$ is some constant function on $M^{3}$.

\section{Pseudosymmetric contact metric 3-manifolds with $\nabla_{\xi} \tau=0$}

Theorem 4.1. Let $M^{3}$ be a 3-dimensional pseudosymmetric contact metric manifold satisfying $\nabla_{\xi} \tau=0$. Then $M^{3}$ is of constant type, and it is either Sasakian, flat, or locally isometric to either $\mathrm{SU}(2)$ or $\mathrm{SL}(2, R)$, where these two Lie groups are equipped with a left invariant metric.

Proof. We consider the open subsets

$$
\begin{aligned}
U_{0} & =\{p \in M: \lambda=0 \text { in a neighborhood of } p\}, \\
U & =\{p \in M: \lambda \neq 0 \text { in a neighborhood of } p\}
\end{aligned}
$$

of $M$. Suppose $M=U_{0}$, that is, $(\xi, \eta, \phi, g)$ is a Sasakian structure. In [2005], Cho and Inoguchi proved that $M$ is a pseudosymmetric space of constant type.

If $U$ is not empty, let $\{e, \phi e, \xi\}$ be a $\phi$-basis. In contact metric 3-manifolds, the assumption $\nabla_{\xi} \tau=0$ is equivalent to $a=Z=0$; see [Gouli-Andreou and Xenos 1998a]. Hence (3-1) becomes

$$
\begin{aligned}
A B & =0 & & \\
B(D-L) & =0, & A^{2}+D(D-C) & =L(D-C), \\
A(D-L) & =0, & B^{2} & =A^{2},
\end{aligned}
$$

or equivalently

$$
\begin{gathered}
Z=A=B=a=0, \\
(D-L)(D-C)=0,
\end{gathered}
$$

where the functions $A, B, C, D, I$ and $Z$ are given by (2-12) and (2-16). Using Proposition 2.4 we obtain $Q \phi=\phi Q$, and hence the manifold is pseudosymmetric of constant type (by [Cho and Inoguchi 2005] and Proposition 3.3). The equation $(D-L)(D-C)=0$ does not contribute any further information to our problem, and hence the proof is completed by [Blair et al. 1990, Theorem 3.3] and [Blair and Chen 1992, Main Theorem].

\section{Pseudosymmetric contact metric 3-manifolds of constant type with $\nabla_{\xi} \tau=2 a \tau \phi$}

Theorem 5.1. Let $M^{3}$ be a 3-dimensional pseudosymmetric contact metric manifold of constant type satisfying the condition $\nabla_{\xi} \tau=2 a \tau \phi$, where $a$ is a smooth function. Then the functions $a$ and $\operatorname{Tr} l$ are constant on $M^{3}$, and $M^{3}$ is either 
- Sasakian;

- flat;

- locally isometric to either $\mathrm{SU}(2)$ or $\mathrm{SL}(2, R)$, where both Lie groups are equipped with a left invariant metric;

- semi-K contact of constant type $L=-4$, with $\operatorname{Tr} l=0$ and with constant scalar curvature $r=-4$;

- semi-K contact of constant type $L=a^{2}$, with $\operatorname{Tr} l=-2 a(2+a)$;

- of constant scalar curvature $r=4 a$ and of constant type $L=2 a$, with $\operatorname{Tr} l=0$;

- of constant scalar curvature $r=2 a(4-a)$ and of constant type $L=a^{2}$, with $\operatorname{Tr} l=2 a(2-a)$

- of constant scalar curvature $r=2 a(4-a)$ and of constant type $L=\operatorname{Tr} l-a^{2}$, with $\operatorname{Tr} l=2 a(2-a)$.

Proof. We consider again the open subsets of (4-1). If $M=U_{0}$, then $M$ is Sasakian and hence it is a pseudosymmetric space of constant type [Cho and Inoguchi 2005]. Next, assume that $U$ is not empty, and let $\{e, \phi e, \xi\}$ be a $\phi$-basis. Using (2-10) and (2-11), we can prove that the assumption $\nabla_{\xi} \tau=2 a \tau \phi$ is equivalent to $Z=\xi \cdot \lambda=0$, and hence the system (3-1) becomes

$$
\begin{gathered}
A B=0, \\
D B=L B,
\end{gathered}
$$

or using (2-16), it is

$$
\begin{aligned}
& 0=A B, \\
& 0=B\left(2 a \lambda-\lambda^{2}+1-L\right), \\
& 0=A\left(-2 a \lambda-\lambda^{2}+1-L\right), \\
& 0=A^{2}+\left(-2 a \lambda-2 \lambda^{2}+2+b^{2}+c^{2}-2 a-(e \cdot c)-(\phi e \cdot b)\right) \\
& \quad \times\left(2 a \lambda-\lambda^{2}+1-L\right), \\
& 0=B^{2}+\left(2 a \lambda-2 \lambda^{2}+2+b^{2}+c^{2}-2 a-(e \cdot c)-(\phi e \cdot b)\right) \\
& 0=Z=\xi \cdot \lambda \quad \times\left(-2 a \lambda-\lambda^{2}+1-L\right),
\end{aligned}
$$

To study this system we consider these open subsets of $U$ :

$$
\begin{aligned}
& U^{\prime}=\{p \in U: A=2 b \lambda-(\phi e \cdot \lambda)=0 \text { in a neighborhood of } p\}, \\
& U_{3}=\{p \in U: A \neq 0 \text { in a neighborhood of } p\}
\end{aligned}
$$


where $U^{\prime} \cup U_{3}$ is open and dense in the closure of $U$. In $U^{\prime}$, we have

$$
\begin{aligned}
& 0=\left(-2 a \lambda-2 \lambda^{2}+2+b^{2}+c^{2}-2 a-(e \cdot c)-(\phi e \cdot b)\right)\left(2 a \lambda-\lambda^{2}+1-L\right), \\
& 0=B\left(2 a \lambda-\lambda^{2}+1-L\right), \\
& 0=B^{2}+\left(2 a \lambda-2 \lambda^{2}+2+b^{2}+c^{2}-2 a-(e \cdot c)-(\phi e \cdot b)\right) \\
& 0=Z=\xi \cdot \lambda .
\end{aligned}
$$

We consider these open subsets of $U^{\prime}$ :

$$
\begin{aligned}
& U_{1}=\left\{p \in U^{\prime}: B=2 c \lambda-(e \cdot \lambda)=0 \text { in a neighborhood of } p\right\}, \\
& U_{2}=\left\{p \in U^{\prime}: B \neq 0 \text { in a neighborhood of } p\right\},
\end{aligned}
$$

where $U_{1} \cup U_{2}$ is open and dense in the closure of $U^{\prime}$. Because $B \neq 0$ in $U_{2}$, we have $2 a \lambda-\lambda^{2}+1-L=0$ there. Hence $U_{1}$ can also be described as the set of $p \in U$ satisfying

$$
\begin{aligned}
& 0=2 b \lambda-(\phi e \cdot \lambda), \quad 0=2 c \lambda-(e \cdot \lambda)=0, \quad 0=\xi \cdot \lambda, \\
& 0=\left(2 a \lambda-2 \lambda^{2}+2+b^{2}+c^{2}-2 a-(e \cdot c)-(\phi e \cdot b)\right)\left(-2 a \lambda-\lambda^{2}+1-L\right), \\
& 0=\left(-2 a \lambda-2 \lambda^{2}+2+b^{2}+c^{2}-2 a-(e \cdot c)-(\phi e \cdot b)\right)\left(2 a \lambda-\lambda^{2}+1-L\right),
\end{aligned}
$$

in a neighborhood of $p$, whereas $U_{2}$ is the set of $p \in U$ satisfying

$$
\begin{aligned}
& 0=2 b \lambda-(\phi e \cdot \lambda), \quad 0=2 a \lambda-\lambda^{2}+1-L, \quad 0=\xi \cdot \lambda, \\
& \begin{aligned}
0 & =\left(2 a \lambda-2 \lambda^{2}+2+b^{2}+c^{2}-2 a-(e \cdot c)-(\phi e \cdot b)\right) \\
& \times\left(-2 a \lambda-\lambda^{2}+1-L\right)+B^{2}
\end{aligned}
\end{aligned}
$$

in a neighborhood of $p$. In $U_{3}$ we have $A \neq 0$ (or equivalently $B=2 c \lambda-(e \cdot \lambda)=0$ ) and the system becomes

$$
\begin{aligned}
& 0=-2 a \lambda-\lambda^{2}+1-L, \\
& 0=A^{2}+\left(-2 a \lambda-2 \lambda^{2}+2+b^{2}+c^{2}-2 a-(e \cdot c)-(\phi e \cdot b)\right) \\
& 0=Z=\xi \cdot \lambda .
\end{aligned}
$$

The set $U_{3}$ is also described as the set of $p \in U$ for which there is a neighborhood satisfying

$$
\begin{aligned}
& \begin{array}{l}
0=2 c \lambda-(e \cdot \lambda), \quad 0=-2 a \lambda-\lambda^{2}+1-L, \quad 0=\xi \cdot \lambda, \\
0=A^{2}+\left(-2 a \lambda-2 \lambda^{2}+2+b^{2}+c^{2}-2 a-(e \cdot c)-(\phi e \cdot b)\right) \\
\times\left(2 a \lambda-\lambda^{2}+1-L\right) .
\end{array}
\end{aligned}
$$

We shall study the initial system at each $U_{i}$ for $i=1,2,3$. 
In $U_{1}$, we have

$(5-1)(\phi e \cdot \lambda)=2 b \lambda$,

$(5-2) \quad(e \cdot \lambda)=2 c \lambda$

$$
\begin{aligned}
& 0=\xi \cdot \lambda, \\
& 0=\left(2 a \lambda-2 \lambda^{2}+2+b^{2}+c^{2}-2 a-(e \cdot c)-(\phi e \cdot b)\right) \\
& \times\left(-2 a \lambda-\lambda^{2}+1-L\right), \\
& 0=\left(-2 a \lambda-2 \lambda^{2}+2+b^{2}+c^{2}-2 a-(e \cdot c)-(\phi e \cdot b)\right) \\
& \times\left(2 a \lambda-\lambda^{2}+1-L\right) \text {. }
\end{aligned}
$$

Differentiating the equations (5-1) and (5-2) with respect to $e$ and $\phi e$, respectively, and subtracting we get $[e, \phi e] \lambda=2 b(e \cdot \lambda)+2 \lambda(e \cdot b)-2 c(\phi e \cdot \lambda)-2 \lambda(\phi e \cdot c)$ or, because of (2-13), (5-1), (5-2) and (5-3),

$$
e \cdot b=\phi e \cdot c .
$$

Differentiating the equations (5-1), (5-3) with respect to $\xi$ and $\phi e$, respectively, and subtracting, we get $[\xi, \phi e] \lambda=2 \lambda(\xi \cdot b)$ or, because of (2-13), (2-17) and (5-2),

$$
\begin{aligned}
& \xi \cdot b=c(\lambda-a-1), \\
& e \cdot a=2 c \lambda .
\end{aligned}
$$

Differentiating the equations (5-2) and (5-3) with respect to $\xi$ and $e$, respectively, and subtracting, we get $[\xi, e] \lambda=2 \lambda(\xi \cdot c)$ or, because of (2-13), (2-17) and (5-1),

$$
\xi \cdot c=b(\lambda+a+1),
$$

$$
\phi e \cdot a=-2 b \lambda \text {. }
$$

In order to study the system of (5-4) and (5-5) we consider these open subsets of $U_{1}$ :

$$
\begin{array}{r}
V=\left\{p \in U_{1}: 2 a \lambda-2 \lambda^{2}+2+b^{2}+c^{2}-2 a-(e \cdot c)-(\phi e \cdot b)=0\right. \\
\text { in a neighborhood of } p\}, \\
V^{\prime}=\left\{p \in U_{1}: 2 a \lambda-2 \lambda^{2}+2+b^{2}+c^{2}-2 a-(e \cdot c)-(\phi e \cdot b) \neq 0\right. \\
\text { in a neighborhood of } p\},
\end{array}
$$

where $V \cup V^{\prime}$ is open and dense in the closure of $U_{1}$. In the set $V$, the Equation (5-5) also holds; hence we consider these open subsets of $V$ :

$$
\begin{array}{r}
V_{1}=\left\{p \in V: \quad-2 a \lambda-2 \lambda^{2}+2+b^{2}+c^{2}-2 a-(e \cdot c)-(\phi e \cdot b)=0,\right. \\
2 a \lambda-2 \lambda^{2}+2+b^{2}+c^{2}-2 a-(e \cdot c)-(\phi e \cdot b)=0 \\
\text { in a neighborhood of } p\}
\end{array}
$$


and

$$
\begin{gathered}
V_{2}=\left\{p \in V: 2 a \lambda-2 \lambda^{2}+2+b^{2}+c^{2}-2 a-(e \cdot c)-(\phi e \cdot b)=0,\right. \\
\left.2 a \lambda-\lambda^{2}+1-L=0 \quad \text { in a neighborhood of } p\right\},
\end{gathered}
$$

where $V_{1} \cup V_{2}$ is open and dense in the closure of $V$. Similarly for $V^{\prime}$, where $-2 a \lambda-\lambda^{2}+1-L=0$, we consider the open subsets

$$
\begin{aligned}
& V_{3}=\left\{p \in V^{\prime}:-2 a \lambda-2 \lambda^{2}+2+b^{2}+c^{2}-2 a-(e \cdot c)-(\phi e \cdot b)=0,\right. \\
& \left.-2 a \lambda-\lambda^{2}+1-L=0 \quad \text { in a neighborhood of } p\right\}, \\
& V_{4}=\left\{p \in V^{\prime}:-2 a \lambda-\lambda^{2}+1-L=0,\right. \\
& \left.2 a \lambda-\lambda^{2}+1-L=0 \quad \text { in a neighborhood of } p\right\},
\end{aligned}
$$

where $V_{3} \cup V_{4}$ is open and dense in the closure of $V^{\prime}$ and the set $U V_{i}$ is open and dense in the closure of $U_{1}$. We shall prove that the functions $\lambda$ and $a$ are constant at every $V_{i}$ for $i=1,2,3,4$

Now

$$
\text { in } V_{1}, \quad\left\{\begin{array}{r}
-2 a \lambda-2 \lambda^{2}+2+b^{2}+c^{2}-2 a-(e \cdot c)-(\phi e \cdot b)=0, \\
2 a \lambda-2 \lambda^{2}+2+b^{2}+c^{2}-2 a-(e \cdot c)-(\phi e \cdot b)=0 .
\end{array}\right.
$$

Subtracting these two equations, we deduce that $a=0$ in $V_{1} \subset U$. Hence from (5-8) and (5-10) we have $c=b=0$, and from (5-1) and (5-2) we have $\phi e \cdot \lambda=e \cdot \lambda=0$. These, together with (5-3), give $\lambda=$ constant in $V_{1}$. Moreover, if we put $a=b=c=0$ in one of the equations of the set $V_{1}$, we finally get $\lambda^{2}=1$ and the structure is flat.

Next,

$(5-11) \quad$ in $V_{2} \quad\left\{\begin{array}{l}2 a \lambda-2 \lambda^{2}+2+b^{2}+c^{2}-2 a-(e \cdot c)-(\phi e \cdot b)=0, \\ 2 a \lambda-\lambda^{2}+1-L=0,\end{array}\right.$

(5-12) in $V_{3} \quad\left\{\begin{array}{l}-2 a \lambda-2 \lambda^{2}+2+b^{2}+c^{2}-2 a-(e \cdot c)-(\phi e \cdot b)=0, \\ -2 a \lambda-\lambda^{2}+1-L=0 .\end{array}\right.$

In $V_{2}$ we differentiate the equation $2 a \lambda-\lambda^{2}+1-L=0$ with respect to $\xi$, $\phi e$ and $e$, and because of (5-3), (5-8), (5-10) we obtain respectively

$$
\begin{aligned}
\xi \cdot a & =0, \\
b(a-2 \lambda) & =0, \\
a c & =0,
\end{aligned}
$$

while in $V_{3}$ from $-2 a \lambda-\lambda^{2}+1-L=0$ we obtain Equation (5-13), and

$$
b a=0, \quad c(a+2 \lambda)=0 .
$$


Differentiating the relations (5-8) and (5-13) with respect to $\xi$ and $e$, respectively, and subtracting, we get $[\xi, e] a=2 \lambda(\xi \cdot c)$ or, because of (2-13), (5-9) and (5-10),

$$
b(\lambda+a+1)=0 .
$$

Similarly, differentiating (5-10) and (5-13) with respect to $\xi$ and $\phi e$, respectively, and subtracting, we have $[\xi, \phi e] a=-2 \lambda(\xi \cdot b)$ or, because of (2-13), (5-7) and (5-8),

$$
c(\lambda-a-1)=0 .
$$

To study the system of (5-17) and (5-18), we consider these open subsets of $V_{2}$ :

$$
\begin{aligned}
G & =\left\{p \in V_{2}: b=0 \text { in a neighborhood of } p\right\}, \\
G^{\prime} & =\left\{p \in V_{2}: b \neq 0 \text { in a neighborhood of } p\right\},
\end{aligned}
$$

where $G \cup G^{\prime}$ is open and dense in the closure of $V_{2}$.

In $G$ we have $c(\lambda-a-1)=0$, hence we consider these open subsets of $G$ :

$$
\begin{aligned}
& G_{1}=\{p \in G: c=0 \text { in a neighborhood of } p\}, \\
& G_{2}=\{p \in G: c \neq 0 \text { in a neighborhood of } p\},
\end{aligned}
$$

where $G_{1} \cup G_{2}$ is open and dense in the closure of $G$. These sets are described more specifically as

$$
\begin{aligned}
& G_{1}=\left\{p \in G \subset V_{2}: b=c=0 \text { in a neighborhood of } p\right\}, \\
& G_{2}=\left\{p \in G \subset V_{2}: b=\lambda-a-1=0 \text { in a neighborhood of } p\right\} .
\end{aligned}
$$

The set $G^{\prime}$ (where $b \neq 0$ or equivalently $\lambda+a+1=0$ ) is decomposed similarly as

$$
\begin{aligned}
& G_{3}=\left\{p \in G^{\prime}: c=0 \text { in a neighborhood of } p\right\}, \\
& G_{4}=\left\{p \in G^{\prime}: c \neq 0 \text { in a neighborhood of } p\right\},
\end{aligned}
$$

where $G_{3} \cup G_{4}$ is open and dense in the closure of $G^{\prime}$. These can also be written

$$
\begin{aligned}
& G_{3}=\left\{p \in G^{\prime} \subset V_{2}: c=\lambda+a+1=0 \text { in a neighborhood of } p\right\}, \\
& G_{4}=\left\{p \in G^{\prime} \subset V_{2}: \lambda+a+1=\lambda-a-1=0 \text { in a neighborhood of } p\right\} .
\end{aligned}
$$

We have $V_{2} \subset U$ where $\lambda \neq 0$; hence $G_{4}=\varnothing$.

In $G_{1}, b=c=0$. From (5-1), (5-2), (5-3), (5-8), (5-10) and (5-13), we can conclude that $\lambda$ and $a$ are constant in $G_{1}$, and from the first of (5-11), we have $(\lambda-1)(a-\lambda-1)=0$. We consider these open subsets of $G_{1}$ :

$$
\begin{aligned}
& K_{1}=\left\{p \in G_{1}: \lambda=1 \text { in a neighborhood of } p\right\}, \\
& K_{2}=\left\{p \in G_{1}: \lambda \neq 1 \text { in a neighborhood of } p\right\} .
\end{aligned}
$$

In $K_{1}$, we get $\operatorname{Tr} l=0, L=2 a$, and, from (2-19), $r=4 a$. In $K_{2}$, we have $a-\lambda-1=0, \operatorname{Tr} l=2 a(2-a), L=a^{2}$, and $r=2 a(4-a)$. 
In $G_{2}, b=\lambda-a-1=0$. Using this to eliminate $a$ from the second equation of (5-11), we obtain $\lambda^{2}-2 \lambda+1-L=0$. We suppose that there is a point $p$ in $G_{2}$ at which $e \cdot \lambda \neq 0$. Then there is a neighborhood $S$ of $p$ in which $e \cdot \lambda \neq 0$. We differentiate $\lambda^{2}-2 \lambda+1-L=0$ with respect to $e$ twice and obtain $e \cdot \lambda=0$ in $S$, a contradiction. Hence $e \cdot \lambda=0$ (and $c=0$ ), and from (5-1) and (5-3), we conclude that $\lambda$ is constant in $G_{2}$ and similarly $a=\lambda-1$. In particular, from (5-11), we obtain $\lambda=1$ and $a=0$; hence the structure is flat.

We have proved that $\lambda$ and $a$ are constant on $G_{1}$ and $G_{2}$. The set $G_{1} \cup G_{2}$ is open and dense in the closure of $G$. Hence $\lambda$ and $a$ are constant everywhere in $G$.

In $G_{3}, c=\lambda+a+1=0$. Using this to eliminate $a$ from the second equation of (5-11), we obtain $-3 \lambda^{2}-2 \lambda+1-L=0$. If we assume that there is a point $p$ in $G_{3}$ at which $\phi e \cdot \lambda \neq 0$, then there is a neighborhood $S$ of $p$ in which $\phi e \cdot \lambda \neq 0$. We differentiate $-3 \lambda^{2}-2 \lambda+1-L=0$ with respect to $\phi e$ twice and obtain $\phi e \cdot \lambda=0$ in $S$, a contradiction. Thus $\phi e \cdot \lambda=0$ everywhere in $G_{3}$, which gives $b=0$. We note that $\xi \cdot \lambda=\phi e \cdot \lambda=e \cdot \lambda=0$, so $\lambda$ is constant in $G_{3}$, and obviously $a=-\lambda-1$. Moreover, if we put $b=c=0$ and $a=-\lambda-1$ in the system (5-11), we get $\lambda^{2}=1$ (hence $\operatorname{Tr} l=0$ ), $a=0$ or -2 , and $L=-2 a(a+1)$. If $a=0$, we obtain a flat structure, while if $a=-2$, we have a semi-K contact structure with constant scalar curvature $r=4 a=-8$.

The functions $\lambda$ and $a$ are constant in $G$ and $G^{\prime}$. The set $G \cup G^{\prime}$ is open and dense in the closure of $V_{2}$; hence $\lambda$ and $a$ are constant in $V_{2}$, and Equations (5-14) and (5-15) are satisfied because $b=c=0$.

We similarly consider these open subsets of $V_{3}$ :

$$
\begin{aligned}
& G_{1}^{\prime}=\left\{p \in V_{3}: b=c=0 \text { in a neighborhood of } p\right\}, \\
& G_{2}^{\prime}=\left\{p \in V_{3}: b=\lambda-a-1=0 \text { in a neighborhood of } p\right\}, \\
& G_{3}^{\prime}=\left\{p \in V_{3}: c=\lambda+a+1=0 \text { in a neighborhood of } p\right\}, \\
& G_{4}^{\prime}=\left\{p \in V_{3}: \lambda+a+1=\lambda-a-1=0 \text { in a neighborhood of } p\right\} .
\end{aligned}
$$

The set $\bigcup G_{i}^{\prime}$ is open and dense subset of $V_{3}$ and $G_{4}^{\prime}=\varnothing$.

In $G_{1}^{\prime}, \quad b=c=0$. From (5-1), (5-2), (5-3), (5-8), (5-10) and (5-13) we can conclude that $\lambda$ and $a$ are constant in $G_{1}^{\prime}$. From the first of (5-12) we have $(\lambda+1)(a+\lambda-1)=0$. We consider these open subsets of $G_{1}^{\prime}$ :

$$
\begin{aligned}
& K_{1}^{\prime}=\left\{p \in G_{1}^{\prime}: \lambda=-1 \text { in a neighborhood of } p\right\}, \\
& K_{2}^{\prime}=\left\{p \in G_{1}^{\prime}: \lambda \neq-1 \text { in a neighborhood of } p\right\} .
\end{aligned}
$$

In $K_{1}^{\prime}$, we get $\operatorname{Tr} l=0, L=2 a$, and, from (2-19), $r=4 a$. In $K_{2}^{\prime}$ we have $a+\lambda-1=0, \operatorname{Tr} l=2 a(2-a), L=a^{2}$, and $r=2 a(4-a)$.

In $G_{2}^{\prime}, b=\lambda-a-1=0$. Using this to eliminate $a$ from the second equation of (5-12), we obtain $-3 \lambda^{2}+2 \lambda+1-L=0$. If we assume $e \cdot \lambda \neq 0$, we may differentiate 
this equation twice with respect to $e$, obtaining $e \cdot \lambda=0$, a contradiction. Hence $e \cdot \lambda=0$ (and $c=0$ ), and from (5-1) and (5-3) we can conclude that $\lambda$ is constant in $G_{2}^{\prime}$ and similarly $a=\lambda-1$. In particular, from the system (5-12), we have $\lambda^{2}=1$ (hence $\operatorname{Tr} l=0), a=0$ or -2 , and $L=-2 a(a+1)$. If $a=0$ we obtain a flat structure, and if $a=-2$ we have a semi-K contact structure with constant scalar curvature $r=4 a=-8$.

In $G_{3}^{\prime}, c=\lambda+a+1=0$. Using this to eliminate $a$ from the second equation of $V_{3}$, we get $\lambda^{2}+2 \lambda+1-L=0$. If we assume $\phi e \cdot \lambda \neq 0$, we may differentiate this equation with twice respect to $\phi e$, obtaining $\phi e \cdot \lambda=0$, a contradiction. Thus $\phi e \cdot \lambda=0$ everywhere in $G_{3}^{\prime}$, which gives $b=0$. We note that $\xi \cdot \lambda=\phi e \cdot \lambda=e \cdot \lambda=0$. Thus $\lambda$ is constant in $G_{3}^{\prime}$, and obviously $a=-\lambda-1$. Moreover, if we put $b=c=0$ and $a=-\lambda-1$ in the system (5-12), we get $\lambda=-1$ and $a=0$; hence the structure is flat.

As in case of $V_{2}$, we have that the functions $\lambda$ and $a$ are constant in $V_{3}$, and the equations (5-16) are satisfied because $b=c=0$.

In $V_{4}, 2 a \lambda-\lambda^{2}+1-L=0$ and $-2 a \lambda-\lambda^{2}+1-L=0$. Working as in the set $V_{1}$, we find that $a=0$ (hence Theorem 4.1 applies), $b=c=0$, and $\lambda$ is constant in $V_{4}$, that is, $\lambda^{2}=1-L \geq 0$. Also, from (2-19), $r=\operatorname{Tr} l=2 L$.

The functions $\lambda$ and $a$ are constant in each $V_{i}$ for $i=1,2,3,4$, and the set $\bigcup V_{i}$ is open and dense in the closure of $U_{1}$. Hence $\lambda$ and $a$ are constant in $U_{1}$.

In $U_{2}$,

$$
\begin{aligned}
\phi e \cdot \lambda & =2 b \lambda, \\
0 & =\xi \cdot \lambda, \\
0 & =2 a \lambda-\lambda^{2}+1-L, \\
0 & =B^{2}+\left(2 a \lambda-2 \lambda^{2}+2+b^{2}+c^{2}-2 a-(e \cdot c)-(\phi e \cdot b)\right) \\
& \times\left(-2 a \lambda-\lambda^{2}+1-L\right) .
\end{aligned}
$$

We differentiate (5-19) with respect to $\xi, \phi e$, and $e$ and because of (5-1) and (5-3) we obtain respectively

$$
\begin{aligned}
0 & =\xi \cdot a, \\
\phi e \cdot a & =2 b \lambda-2 a b, \\
0 & =(a-\lambda)(e \cdot \lambda)+\lambda(e \cdot a)=0 .
\end{aligned}
$$

We differentiate Equations (5-1) and (5-3) with respect to $\xi$ and $\phi e$ respectively and subtract. Then, because of (5-3), (2-13) and (2-17), we obtain

$$
(3 \lambda-a-1)(e \cdot \lambda)-2 \lambda(e \cdot a)=2 \lambda c(\lambda-a-1)
$$


We consider the system of (5-23) and (5-24) with unknown functions the derivatives of $e \cdot \lambda$ and $e \cdot a$. We consider these open subsets of $U_{2}$ :

$$
\begin{aligned}
& F_{1}=\left\{p \in U_{2} \subset U:-a-\lambda+1 \neq 0 \text { in a neighborhood of } p\right\}, \\
& F_{2}=\left\{p \in U_{2} \subset U:-a-\lambda+1=0 \text { in a neighborhood of } p\right\} .
\end{aligned}
$$

In the neighborhood $F_{1}$ solving the system of (5-23) and (5-24), we obtain

$$
(-a-\lambda+1)(e \cdot \lambda)=-2 \lambda c(\lambda-a-1) .
$$

From (2-17), (5-1) and (5-22), we obtain

$$
\xi \cdot c=-b(3 \lambda-3 a-1)
$$

We differentiate (5-3), (5-25) with respect to $e$ and $\xi$, respectively, and subtract. Then using (5-3), (5-21), we obtain $(-a-\lambda+1)[e, \xi] \lambda=2 \lambda(\lambda-a-1)(\xi \cdot c)$ or, by (2-13), (5-1), (5-26), and $\lambda \neq 0$ (as $F_{1} \subset U$ ), we finally obtain

$$
b\left(a^{2}+\lambda^{2}-a \lambda+a-\lambda\right)=0
$$

We work in the open subset $F_{1}$ and suppose that there is a point $p$ in $F_{1}$ at which $b \neq 0$ (or equivalently, by (5-1), $\phi e \cdot \lambda \neq 0$ ). The function $b$ is smooth, and because of its continuity there is an open neighborhood $S$ of $p$ such that $S \subset F_{1}$ and $b \neq 0$ everywhere in $S$. Hence from (5-27) we have in $S$

$$
a^{2}+\lambda^{2}-a \lambda+a-\lambda=0 .
$$

We differentiate this equation with respect to $\phi e$, use (5-1) and (5-22), and with the assumption $b \neq 0$ obtain $2 a^{2}-2 a \lambda-\lambda^{2}+a=0$. This last equation together with (5-28) gives $-3 \lambda^{2}-a+2 \lambda=0$. We differentiate this equation with respect to $\phi e$. Then using (5-1) and (5-22), we get $2 b\left(-6 \lambda^{2}+a+\lambda\right)=0$ or equivalently $-6 \lambda^{2}+a+\lambda=0$. The equations $-3 \lambda^{2}-a+2 \lambda=0$ and $-6 \lambda^{2}+a+\lambda=0$ give $-9 \lambda^{2}+3 \lambda=0$. We differentiate this equation twice with respect to $\phi e$ and obtain $\phi e \cdot \lambda=0$ or equivalently $b=0$ everywhere in $S$, a contradiction. Hence, from (5-27) we deduce that $b=0$ everywhere in $F_{1}$.

Equation (5-1) and $b=0$ give $\phi e \cdot \lambda=0$, which together with (5-3) gives $[\xi, \phi e] \lambda=0$, or because of $(2-13),(\lambda-a-1)(e \cdot \lambda)=0$. Let's suppose that there is a point $q \in F_{1}$ at which $e \cdot \lambda \neq 0$. Then, there is a neighborhood $Y$ of $q$ in which $e \cdot \lambda \neq 0$. In $Y$ we then have $\lambda-a-1=0$, and hence from (5-25) we have $(-a-\lambda+1)(e \cdot \lambda)=0$, which in $Y \subset F_{1}$ gives $e \cdot \lambda=0$, a contradiction. Hence $e \cdot \lambda=0$ everywhere in $F_{1}$. Then $\xi \cdot \lambda=\phi e \cdot \lambda=e \cdot \lambda=0$ implies that $\lambda$ is constant in $F_{1}$. From (5-21), (5-22) and (5-23) we obtain that $a$ is constant in $F_{1}$ and from 
(5-25) we have $c(\lambda-a-1)=0$. We consider these two open subsets of $F_{1}$ :

$$
\begin{aligned}
& J_{1}=\left\{p \in F_{1}: c=0 \text { in a neighborhood of } p\right\}, \\
& J_{2}=\left\{p \in F_{1}: c \neq 0 \text { in a neighborhood of } p\right\} .
\end{aligned}
$$

In $J_{1}$, Equation (5-20) yields $a(\lambda-1)(a-\lambda-1)=0$, where $a$ and $\lambda$ are constant and $b=0$. We consider these two open subsets of $J_{1}$ :

$$
\begin{aligned}
& H_{1}=\left\{p \in J_{1}: a=0 \text { in a neighborhood of } p\right\}, \\
& H_{2}=\left\{p \in J_{1}: a \neq 0 \text { in a neighborhood of } p\right\} .
\end{aligned}
$$

In $H_{1}$, we have $\nabla_{\xi} \tau=0$ (hence Theorem 4.1 applies) and from (2-19) $r=2 L$. In $H_{2}$ we have $(\lambda-1)(a-\lambda-1)=0$, and hence we consider these two open subsets of $\mathrm{H}_{2}$ :

$$
\begin{aligned}
& H_{3}=\left\{p \in J_{1}: \lambda=1 \text { in a neighborhood of } p\right\}, \\
& H_{4}=\left\{p \in J_{1}: \lambda \neq 1 \text { in a neighborhood of } p\right\}
\end{aligned}
$$

In $H_{3}$, we have $\operatorname{Tr} l=0, L=2 a$ by (5-19), and $r=4 a$ by (2-19). In $H_{4}$ we obtain $a-\lambda-1=0, \operatorname{Tr} l=2 a(2-a), L=a^{2}$, and $r=2 a(4-a)$.

In $J_{2}$, we have $b=a-\lambda+1=0$ (a semi-K contact structure with constant $a$ and $\lambda)$; hence $\operatorname{Tr} l=-2 a(2+a), L=a^{2}$ by (5-19), and $c^{2}+a(e \cdot c)+4 a^{2}=0$ by (5-20). The set $J_{1} \cup J_{2}$ is open and dense inside the closure of $F_{1}$; hence we conclude that $b=0$ and that $a$ and $\lambda$ are constant in $F_{1}$.

In the open set $F_{2}$ we have $-a-\lambda+1=0$, which together with (5-19) gives $-3 \lambda^{2}+2 \lambda+1-L=0$. If we assume $e \cdot \lambda \neq 0$, we may differentiate this equation twice with respect to $e$ and obtain $e \cdot \lambda=0$, a contradiction. Hence $e \cdot \lambda=0$. Similarly we can deduce that $\phi e \cdot \lambda=0$ (so (5-1) implies $b=0$ ), and hence $\lambda$ is constant in $F_{2}$. Obviously $a=-\lambda+1$ is constant in $F_{2}$. The system of (5-23) and (5-24) gives $c a=0$. We consider these two open subsets of $F_{2}$ :

$$
\begin{aligned}
& Q_{1}=\left\{p \in F_{2}: c=0 \text { in a neighborhood of } p\right\}, \\
& Q_{2}=\left\{p \in F_{2}: c \neq 0 \text { in a neighborhood of } p\right\} .
\end{aligned}
$$

In $Q_{1}$, (5-19) implies $L=\operatorname{Tr} l-a^{2}$, where $\operatorname{Tr} l=2 a(2-a)$ and $r=2 a(4-a)$. In $Q_{2}$, we have a 3- $\tau$ manifold structure with $\operatorname{Tr} l=L=0$.

We have proved that $\lambda$ and $a$ are constant in $F_{1}$ and $F_{2}$. Since $F_{1} \cup F_{2}$ is open and dense inside the closure of $U_{2}$, we conclude that $\lambda$ and $a$ are constant in $U_{2}$. 
In $U_{3}$,

$$
\begin{aligned}
2 c \lambda & =(e \cdot \lambda), \\
0 & =\xi \cdot \lambda, \\
0 & =-2 a \lambda-\lambda^{2}+1-L, \\
0 & =A^{2}+\left(-2 a \lambda-2 \lambda^{2}+2+b^{2}+c^{2}-2 a\right. \\
& =(e \cdot c)-(\phi e \cdot b)) \\
& \times\left(2 a \lambda-\lambda^{2}+1-L\right) .
\end{aligned}
$$

We differentiate the relation (5-29) with respect to $\xi, e$ and $\phi e$, and because of (5-2) and (5-3) we obtain respectively (5-21),

$$
\begin{aligned}
e \cdot a & =-2 a c-2 c \lambda, \\
0 & =(a+\lambda)(\phi e \cdot \lambda)+\lambda(\phi e \cdot a) .
\end{aligned}
$$

We differentiate (5-2) and (5-3) with respect to $\xi$ and $e$ respectively and subtract. Then by (5-21), we obtain $[\xi, e] \lambda=2 \lambda(\xi \cdot c)$, or because of (2-13) and (2-17),

$$
(3 \lambda+a+1)(\phi e \cdot \lambda)+2 \lambda(\phi e \cdot a)=2 \lambda b(\lambda+a+1) .
$$

Meanwhile from (2-17), (5-2) and (5-31), we obtain

$$
\xi \cdot b=-c(3 a+3 \lambda+1) .
$$

Now consider the system of (5-32) and (5-33) and these two open subsets of $U_{3}$ :

$$
\begin{aligned}
& F_{1}^{\prime}=\left\{p \in U_{3} \subset U: a-\lambda-1 \neq 0 \text { in a neighborhood of } p\right\}, \\
& F_{2}^{\prime}=\left\{p \in U_{3} \subset U: a-\lambda-1=0 \text { in a neighborhood of } p\right\} .
\end{aligned}
$$

In the open set $F_{1}^{\prime}$ of $p$ in which $a-\lambda-1 \neq 0$, we may solve the system of (5-32) and (5-33) to obtain

$$
(a-\lambda-1)(\phi e \cdot \lambda)=-2 \lambda b(\lambda+a+1) .
$$

We differentiate (5-3) and (5-35) with respect to $\phi e$ and $\xi$ respectively and subtract. Then by (5-3) and (5-21) we obtain $(a-\lambda-1)[\phi e, \xi] \lambda=2 \lambda(\lambda+a+1)(\xi \cdot b)$ or, because of (2-13), $(a-\lambda-1)(a-\lambda+1)(e \cdot \lambda)=2 \lambda(\lambda+a+1)(\xi \cdot b)$. Then, using (5-2), (5-34) and $\lambda \neq 0$ (in $F_{1}^{\prime} \subset U$ ), we get

$$
c\left(a^{2}+\lambda^{2}+a \lambda+a+\lambda\right)=0 .
$$

As in the case of Equation (5-27) we can deduce in $F_{1}^{\prime}$ that $c=0$. Equation (5-2), because $c=0$, gives $e \cdot \lambda=0$. This together with (5-3) gives $[e, \xi] \lambda=0$ or, because of $(2-13),(a+\lambda+1)(\phi e \cdot \lambda)=0$. Suppose that there is a point $q \in F_{1}^{\prime}$ at which $\phi e \cdot \lambda \neq 0$. Then there is a neighborhood $S$ of $q$ in which $\phi e \cdot \lambda \neq 0$, and hence $a+\lambda+1=0$. Because $a+\lambda+1=0$, Equation (5-35) gives $(a-\lambda-1)(\phi e \cdot \lambda)=0$. 
Working in $S \subset F_{1}^{\prime}$, where $a-\lambda-1 \neq 0$, we can conclude that $\phi e \cdot \lambda=0$, a contradiction. Hence, $\phi e \cdot \lambda=0$ everywhere in $F_{1}^{\prime}$. In the neighborhood $F_{1}^{\prime}$, we have $\xi \cdot \lambda=\phi e \cdot \lambda=e \cdot \lambda=0$ or equivalently $\lambda$ is constant in $F_{1}^{\prime}$. From (5-21), (5-31) and (5-33), we obtain that $a$ is constant in $F_{1}^{\prime}$, and from (5-32) we have $b(\lambda+a+1)=0$. We consider these two open subsets of $F_{1}^{\prime}$ :

$$
\begin{aligned}
& J_{1}^{\prime}=\left\{p \in F_{1}^{\prime}: b=0 \text { in a neighborhood of } p\right\}, \\
& J_{2}^{\prime}=\left\{p \in F_{1}^{\prime}: b \neq 0 \text { in a neighborhood of } p\right\} .
\end{aligned}
$$

In $J_{1}^{\prime}$, we obtain from (5-30) that $a(\lambda+1)(a+\lambda-1)=0$, where $a$ and $\lambda$ are constant and $c=0$. We consider these two open subsets of $J_{1}^{\prime}$ :

$$
\begin{aligned}
& H_{1}^{\prime}=\left\{p \in J_{1}^{\prime}: a=0 \text { in a neighborhood of } p\right\}, \\
& H_{2}^{\prime}=\left\{p \in J_{1}^{\prime}: a \neq 0 \text { in a neighborhood of } p\right\} .
\end{aligned}
$$

In $H_{1}^{\prime}$, we obtain $\nabla_{\xi} \tau=0$ (hence Theorem 4.1 applies). and $r=2 L$. In $H_{2}^{\prime}$, we have $(\lambda+1)(a+\lambda-1)=0$. We consider these two open subsets of $H_{2}^{\prime}$ :

$$
\begin{aligned}
& H_{3}^{\prime}=\left\{p \in J_{1}: \lambda=-1 \text { in a neighborhood of } p\right\}, \\
& H_{4}^{\prime}=\left\{p \in J_{1}: \lambda \neq-1 \text { in a neighborhood of } p\right\} .
\end{aligned}
$$

In $H_{3}^{\prime}$, we have $\operatorname{Tr} l=0, L=2 a$ by (5-29), and $r=4 a$ by (2-19). In $H_{4}^{\prime}$, we obtain $a+\lambda-1=0, \operatorname{Tr} l=2 a(2-a), L=a^{2}$ and $r=2 a(4-a)$.

In $J_{2}^{\prime}$ we have $c=a+\lambda+1=0$ (a semi-K contact structure with constant $a$ and $\lambda$ ) and hence $\operatorname{Tr} l=-2 a(2+a)$. Then $L=a^{2}$ from (5-29), and from (5-30) we obtain $b^{2}+a(\phi e \cdot b)+4 a^{2}=0$ with $a$ a constant. The set $J_{1}^{\prime} \cup J_{2}^{\prime}$ is open and dense inside the closure of $F_{1}^{\prime}$; hence we can conclude that $c=0$ and that $a$ and $\lambda$ are constant in $F_{1}^{\prime}$.

In the open set $F_{2}^{\prime}$ we have $a-\lambda-1=0$, which together with (5-29) gives $-3 \lambda^{2}-2 \lambda+1-L=0$. If we assume $\phi e \cdot \lambda \neq 0$, we may differentiate this equation twice with respect to $e$, obtaining $\phi e \cdot \lambda=0$, a contradiction. Hence $\phi e \cdot \lambda=0$. Similarly we find that $e \cdot \lambda=0$, and hence $\lambda$ is constant in $F_{2}^{\prime}$. Obviously $a=\lambda+1$ is constant in $F_{2}^{\prime}$. The system of (5-32) and (5-33) gives $b a=0$. We consider these two open subsets of $F_{2}^{\prime}$ :

$$
\begin{aligned}
& Q_{1}^{\prime}=\left\{p \in F_{2}^{\prime}: b=0 \text { in a neighborhood of } p\right\}, \\
& Q_{2}^{\prime}=\left\{p \in F_{2}^{\prime}: b \neq 0 \text { in a neighborhood of } p\right\} .
\end{aligned}
$$

In $Q_{1}^{\prime}$, (5-29) implies $L=\operatorname{Tr} l-a^{2}$, where $\operatorname{Tr} l=2 a(2-a)$ and $r=2 a(4-a)$. In $Q_{2}^{\prime}$, we have a 3- $\tau$ manifold structure with $\operatorname{Tr} l=L=0$. 
We have proved that $\lambda$ and $a$ are constant in $F_{1}^{\prime}$ and $F_{2}^{\prime}$, while $F_{1}^{\prime} \cup F_{2}^{\prime}$ is open and dense inside the closure of $U_{3}$. Hence we conclude that $\lambda$ and $a$ are constant in $U_{3}$.

Finally because $\lambda$ and $a$ are constant in each $U_{i}$ for $i=1,2,3$, and because the set $\bigcup U_{i}$ is open and dense inside of the closure of $U$, we conclude that $\lambda$ and $a$ are constant in $U$. Then by (2-9), $\operatorname{Tr} l=2\left(1-\lambda^{2}\right)$ is also constant in $U$ and obviously on $M^{3}$.

\section{Acknowledgments}

The authors thank Professors R. Deszcz, J. Inoguchi, C. Özgür and Ph. J. Xenos for useful information on pseudosymmetric manifolds. They also thank the referee for useful remarks.

\section{References}

[Belkhelfa et al. 2002] M. Belkhelfa, R. Deszcz, M. Głogowska, M. Hotloś, D. Kowalczyk, and L. Verstraelen, "On some type of curvature conditions", pp. 179-194 in PDEs, submanifolds and affine differential geometry (Warsaw, 2000), edited by B. Opozda et al., Banach Center Publ. 57, Polish Acad. Sci., Warsaw, 2002. MR 2004a:53019 Zbl 1023.53013

[Blair 2002] D. E. Blair, Riemannian geometry of contact and symplectic manifolds, Progress in Mathematics 203, Birkhäuser, Boston, 2002. MR 2002m:53120 Zbl 1011.53001

[Blair and Chen 1992] D. E. Blair and H. Chen, "A classification of 3-dimensional contact metric manifolds with $Q \phi=\phi Q$, II”, Bull. Inst. Math. Acad. Sinica 20:4 (1992), 379-383. MR 94b:53062 Zbl 0767.53023

[Blair et al. 1990] D. E. Blair, T. Koufogiorgos, and R. Sharma, "A classification of 3-dimensional contact metric manifolds with $Q \phi=\phi Q$ ”, Kodai Math. J. 13:3 (1990), 391-401. MR 91j:53015 Zbl 0716.53041

[Boeckx et al. 1996] E. Boeckx, O. Kowalski, and L. Vanhecke, Riemannian manifolds of conullity two, World Scientific Publishing Co., River Edge, NJ, 1996. MR 98h:53075 Zbl 0904.53006

[Calvaruso 2006] G. Calvaruso, "Conformally flat pseudo-symmetric spaces of constant type", Czechoslovak Math. J. 56:131 (2006), 649-657. MR 2007m:53043

[Calvaruso and Perrone 2002] G. Calvaruso and D. Perrone, "Semi-symmetric contact metric threemanifolds”, Yokohama Math. J. 49:2 (2002), 149-161. MR 2003g:53137 Zbl 1047.53017

[Chern and Hamilton 1985] S. S. Chern and R. S. Hamilton, "On Riemannian metrics adapted to three-dimensional contact manifolds", pp. 279-308 in Workshop Bonn 1984, edited by F. Hirzebruch et al., Lecture Notes in Math. 1111, Springer, Berlin, 1985. MR 87b:53060 Zbl 0561.53039

[Cho and Inoguchi 2005] J. T. Cho and J.-I. Inoguchi, "Pseudo-symmetric contact 3-manifolds", J. Korean Math. Soc. 42:5 (2005), 913-932. MR 2006c:53085 Zbl 1081.53018

[Deszcz 1992] R. Deszcz, "On pseudosymmetric spaces”, Bull. Soc. Math. Belg. Sér. A 44:1 (1992), 1-34. MR 96c:53068 Zbl 0808.53012

[Gouli-Andreou and Xenos 1998a] F. Gouli-Andreou and P. J. Xenos, "On 3-dimensional contact metric manifolds with $\nabla_{\xi} \tau=0$ ”, J. Geom. 62:1-2 (1998), 154-165. MR 99e:53035 Zbl 0905.53024

[Gouli-Andreou and Xenos 1998b] F. Gouli-Andreou and P. J. Xenos, "On a class of 3-dimensional contact metric manifolds”, J. Geom. 63:1-2 (1998), 64-75. MR 99k:53091 Zbl 0918.53014 
[Gouli-Andreou et al. 2008] F. Gouli-Andreou, J. Karatsobanis, and P. Xenos, "Conformally flat 3- $\tau$-a manifolds", Differ. Geom. Dyn. Syst. 10 (2008), 107-131. MR 2390006

[Hashimoto and Sekizawa 2000] N. Hashimoto and M. Sekizawa, "Three-dimensional conformally flat pseudo-symmetric spaces of constant type”, Archivum Math. (Brno) 36:4 (2000), 279-286. MR 2001k:53053 Zbl 1054.53060

[Koufogiorgos 1995] T. Koufogiorgos, "On a class of contact Riemannian 3-manifolds", Results Math. 27:1-2 (1995), 51-62. MR 95m:53040 Zbl 0833.53032

[Kowalski and Sekizawa 1996a] O. Kowalski and M. Sekizawa, "Local isometry classes of Riemannian 3-manifolds with constant Ricci eigenvalues $\rho_{1}=\rho_{2} \neq \rho_{3}>0$ ", Archivum Math. (Brno) 32:2 (1996), 137-145. MR 97d:53053 Zbl 0903.53015

[Kowalski and Sekizawa 1996b] O. Kowalski and M. Sekizawa, "Three-dimensional Riemannian manifolds of $c$-conullity two", Chapter 11 of [Boeckx et al. 1996], 1996.

[Kowalski and Sekizawa 1997] O. Kowalski and M. Sekizawa, "Pseudo-symmetric spaces of constant type in dimension three-elliptic spaces", Rendiconti Mat. Appl. (7) 17:3 (1997), 477-512. MR 99a:53032 Zbl 0889.53026

[Kowalski and Sekizawa 1998] O. Kowalski and M. Sekizawa, "Pseudo-symmetric spaces of constant type in dimension three — non-elliptic spaces", Bull. Tokyo Gakugei Univ. (4) 50 (1998), 1-28. MR 99j:53040 Zbl 0945.53020

[Perrone 1990] D. Perrone, "Torsion and critical metrics on contact three-manifolds", Kodai Math. J. 13:1 (1990), 88-100. MR 91b:58038 Zbl 0709.53034

[Szabó 1982] Z. I. Szabó, "Structure theorems on Riemannian spaces satisfying $R(X, Y) \cdot R=0$, I: The local version”, J. Differential Geom. 17:4 (1982), 531-582. MR 84e:53060 Zbl 0508.53025

[Szabó 1985] Z. I. Szabó, "Structure theorems on Riemannian spaces satisfying $R(X, Y) \cdot R=0$, II: Global versions”, Geom. Dedicata 19:1 (1985), 65-108. MR 87c:53099 Zbl 0612.53023

[Takagi 1972] H. Takagi, "An example of Riemannian manifolds satisfying $R(X, Y) \cdot R=0$ but not $\nabla R=$ 0”, Tôhoku Math. J. (2) 24 (1972), 105-108. MR 47 \#7655 Zbl 0237.53041

Received March 3, 2008.

\title{
Florence Gouli-ANDREOU
}

DEPARTMENT OF MATHEMATICS

ARISTOTLE UNIVERSITY OF THESSALONIKI

54124 THESSALONIKI

GREECE

fgouli@math.auth.gr

\author{
EVAGgelia MoutaFi \\ DEPARTMENT OF MATHEMATICS \\ ARISTOTLE UNIVERSITY OF THESSALONIKI \\ 54124 THESSALONIKI \\ GREECE \\ moutafi@sch.gr
}

